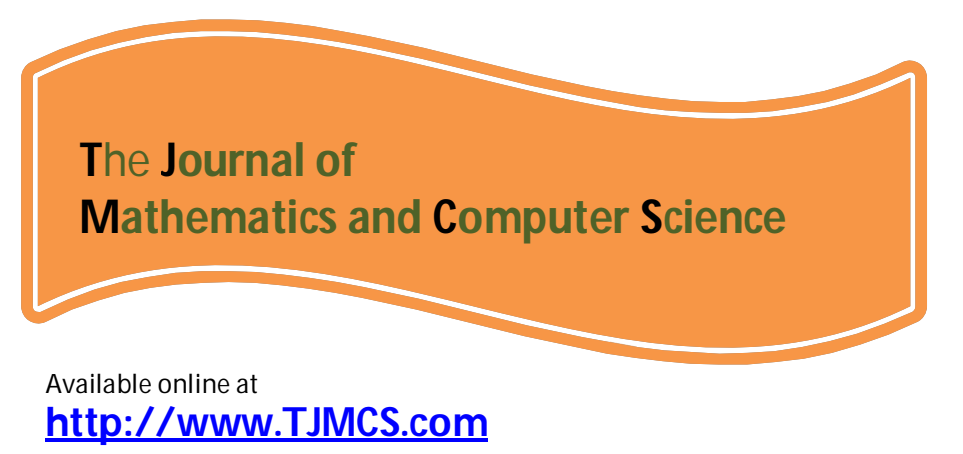

The Journal of Mathematics and Computer Science Vol. 4 No.3 (2012) 295 - 300

\title{
On Lorentzian $\alpha$-Sasakian manifolds
}

\author{
A. Taleshian*,1 and N. Asghari2 \\ 1 Department of Mathematics, University of Mazandaran, \\ P.O.Box 47416-1467, Mazandaran, Iran \\ taleshian@umz.ac.ir \\ 2 Department of Mathematics, University of Mazandaran, \\ P.O.Box 47416-1467, Mazandaran, Iran. \\ nasgharigm2009@yahoo.com
}

Received: February 2012, Revised: May 2012

Online Publication: July 2012

\begin{abstract}
We study Ricci-semi symmetric, $\phi$-Ricci semisymmetric and $\phi$-symmetric Lorentzian $\alpha$ -Sasakian manifolds. Also, we study a Lorentzia $\alpha$-Sasakian manifold satisfies $S(X, \xi) \cdot R=0$.
\end{abstract}

keywords: Ricci semisymmetric Lorentzia $\alpha$-Sasakian manifold, $\phi$-Ricci symmetric Lorentzian $\alpha$-Sasakian manifold, $\phi$-symmetric Lorentzian $\alpha$-Sasakian manifold.

\section{Introduction}

The notion of local symmetry of Rimannian manifolds have been weakened by many authors in several ways to the different extent. As a weaker version of local symmetry, Takahashi [6], introduced the notion of locally $\phi$-symmetry on sasakian manifolds. In respect of contact Geometry, the notion of $\phi$-symmetry was introduced and studied by Boeckx, Buecken and Vanhecke [2], with several examples. In [3], De studied the notion of $\phi$-symmetry with several examples for Kenmotsu manifolds. In 1977, Adati and Matsumoto defined Para-sasakian manifold and special Para-Sasakian manifolds [4], which are special classes of an almost para contact manifold introduced by sato [5].

\footnotetext{
${ }^{*}$ Corresponding author
} 


\section{Preliminaries}

A differentiable manifold $M$ of dimension $n$ is called a Lorentzian $\alpha$-Sasakian manifold if it admits a $(1,1)$ tensor filed $\phi$, a contravariant vector field $\xi$, a covariant vector field $\eta$ and Lorentzian metric $g$ which satisfy $[4,7]$

$$
\begin{aligned}
& \phi^{2}=I+\eta \otimes \xi, \\
& \eta(\xi)=-1, \\
& g(\phi X, \phi Y)=g(X, Y)+\eta(X) \eta(Y), \\
& \phi \xi=0, \eta(\phi X)=0, \\
& g(X, \xi)=\eta(X),
\end{aligned}
$$

for all $X, Y \in T M$. From the above relations it follows that a Lorentzian $\alpha$-Sasakian manifold satisfies

$$
\begin{aligned}
& \nabla_{X} \xi=-\alpha \phi X \\
& \left(\nabla_{X} \eta\right) Y=-\alpha g(X, Y), \\
& \left(\nabla_{X} \phi\right) Y=\alpha g(X, Y) \xi-\alpha \eta(Y) X,
\end{aligned}
$$

where $\nabla$ denotes the operator of covariant differentiation with respect to the Lorentzian metric $g$.

Also, a Lorentzian $\alpha$-Sasakian manifold $M$ is said to be $\eta$-Einstein if its Ricci tensor $S$ is of the form

$$
S(X, Y)=a g(X, Y)+b \eta(X) \eta(Y)
$$

for any vector fields $X, Y$ where $a, b$ are functions on $M$.

Further, on such an From the above relations it follows that a Lorentzian $\alpha$-Sasakian manifold satisfiesthe following relations hold[7]

$$
\begin{aligned}
& R(X, Y) \xi=\alpha^{2}(\eta(Y) X+\eta(X) Y), \\
& R(\xi, X) Y=\alpha^{2}(g(X, Y) \xi+\eta(Y) X), \\
& R(\xi, X) \xi=\alpha^{2}(X+\eta(X) \xi), \\
& S(X, \xi)=(n-1) \alpha^{2} \eta(X), \\
& Q \xi=(n-1) \alpha^{2} \xi, \\
& S(\xi, \xi)=-(n-1) \alpha^{2}, \\
& S(\phi X, \phi Y)=S(X, Y)+(n-1) \alpha^{2} \eta(X) \eta(Y),
\end{aligned}
$$

for any vector fields $X, Y, Z$, where $R(X, Y) Z$ is the curvature tensor, and $S$ is the Ricci tensor.

Definition 2.1 An $n$-dimensional Lorentzian $\alpha$-Sasakian manifold is said to be an Einstein manifold if its Ricci tensor satisfies the condition

$$
S(X, Y)=\lambda g(X, Y)
$$

where $\lambda$ is a constant.

Definition 2.2 A Lorentzian $\alpha$-Sasakian manifold is said to be Ricci-semi symmetric if its Ricci tensor satisfies the condition 


$$
R(X, Y) . S=0
$$

for any vector fields $X, Y$.

\section{Main Results}

In this section, we prove the following theorems:

Theorem 3.1 Let $M$ bean $n$-dimensional Lorentzian $\alpha$-Sasakian manifold. If $M$ is Ricci semisymmetric then it is an $\eta$-Einstein manifold.

Proof. Suppose that M is Ricci semisymmetric then in view of (2.18) we have

$$
R(X, Y) . S=0,
$$

this implies that

$$
S(R(X, Y) U, V)+S(U, R(X, Y) V)=0 .
$$

Putting $X=\xi$ in (3.1) we get

Using (2.11) in (3.2) we get

$$
S(R(\xi, Y) U, V)+S(U, R(\xi, Y) V)=0 .
$$

which implies

$$
S\left(\alpha^{2}(g(Y, U) \xi+\eta(U) Y), V\right)+S\left(U, \alpha^{2}(g(Y, V) \xi+\eta(V) Y)\right)=0,
$$

$$
\begin{aligned}
0=\alpha^{2} g(Y, U) S(\xi, V)+\alpha^{2} \eta(U) S(Y, V) & \\
& +\alpha^{2} g(Y, V) S(U, \xi)+\alpha^{2} \eta(V) S(U, Y),
\end{aligned}
$$

Putting $U=\xi$ in (3.3) and using (2.2), (2.5) and (2.13) we obtain

$$
S(Y, V)=-(n-1) \alpha^{2} g(Y, V)+2(n-1) \alpha^{2} \eta(Y) \eta(V) .
$$

Therefore, in view of (2.9), $M$ is an $\eta$-Einstein manifold. This completes the proof of the theorem.

Definition 3.2 A Lorentzian $\alpha$-Sasakian manifold $M$ is said to be $\phi$-Ricci symmetric if the Ricci operator satisfies

$$
\phi^{2}\left(\left(\nabla_{X} Q\right)(Y)\right)=0
$$

for all vector fields $X$ and $Y$ on $M$ and $S(X, Y)=g(Q X, Y)$ [4].

If $X$ and $Y$ are orthogonal to $\xi$, then manifold is said to be locally $\phi$-Ricci symmetric.

Theorem 3.3 An $n$-dimensional Lorentzian $\alpha$-Sasakian manifold is $\phi$-Ricci symmetric if and only if manifold is an Einstein manifold.

Proof. Suppose that the manifold is $\phi$-Ricci symmetric then in view of Definition 3.2 we have

Using (2.1) in above equation we obtain

$$
\phi^{2}\left(\left(\nabla_{X} Q\right)(Y)\right)=0
$$

$$
\left(\nabla_{X} Q\right)(Y)+\eta\left(\left(\nabla_{X} Q\right)(Y)\right) \xi=0 .
$$

Taking inner product of (3.4) with $Z$ we get

which implies

$$
g\left(\left(\nabla_{X} Q\right)(Y), Z\right)+\eta\left(\left(\nabla_{X} Q\right)(Y)\right) \eta(Z)=0
$$




$$
g\left(\nabla_{X} Q(Y)-Q\left(\nabla_{X} Y\right), Z\right)+\eta\left(\left(\nabla_{X} Q\right)(Y)\right) \eta(Z)=0,
$$

which on simplifying gives

$$
g\left(\nabla_{X} Q(Y), Z\right)-S\left(\nabla_{X} Y, Z\right)+\eta\left(\left(\nabla_{X} Q\right)(Y)\right) \eta(Z)=0 .
$$

Replacing $Y$ by $\xi$ in (3.5) we get

$$
g\left(\nabla_{X} Q(\xi), Z\right)-S\left(\nabla_{X} \xi, Z\right)+\eta\left(\left(\nabla_{X} Q\right)(\xi)\right) \eta(Z)=0 .
$$

Using (2.4), (2.13) and (2.14) in (3.6) we obtain

$$
-(n-1) \alpha^{3} g(\phi X, Z)+\alpha S(\phi X, Z)+\eta\left(\left(\nabla_{X} Q\right)(\xi)\right) \eta(Z)=0 .
$$

Replacing $Z$ by $\phi Z$ in (3.7) we get

$$
S(\phi X, \phi Z)=(n-1) \alpha^{2} g(\phi X, \phi Z) .
$$

Using (2.3) and (2.16) in (3.8) we obtain

$$
S(X, Z)=(n-1) \alpha^{2} g(X, Z) .
$$

Therefore, the manifold is an Einstein manifold.

Next, suppose that the manifold is an Einstein manifold. Then in view of (2.17) we have $S(X, Y)=\lambda g(X, Y)$, wher $S(X, Y)=g(Q X, Y)$ and $\lambda$ is constant. Hence $Q X=\lambda X$. Therefore, we obtain $\phi^{2}\left(\left(\nabla_{X} Q\right)(Y)\right)=0$. This completes the proof.

Theorem 3.4 An $n$-dimensional $(n>3)$, Lorentzian $\alpha$-Sasakian manifold satisfying the condition $S(X, \xi) \cdot R=0$ is an $\eta$-Einstein manifold.

Proof. Since $S(X, \xi) \cdot R=0$ we have

$$
(S(X, \xi) \cdot R)(U, V) Z=0,
$$

which implies

$$
\begin{aligned}
& 0=\left(\left(X \wedge_{S} \xi\right) \cdot R\right)(U, V) Z \\
& =\left(X \wedge_{S} \xi\right) R(U, V) Z+R\left(\left(X \wedge_{S} \xi\right) U, V\right) Z \\
& +R\left(U,\left(X \wedge_{S} \xi\right) V\right) Z+R(U, V)\left(X \wedge_{S} \xi\right) Z,
\end{aligned}
$$

where endomorphism $X \wedge_{S} Y$ is defined by

$$
\left(X \wedge_{S} Y\right) Z=S(Y, Z) X-S(X, Z) Y .
$$

Using (3.10) in (3.9) we get by virtue of (2.13)

$$
\begin{aligned}
0=(n & -1) \alpha^{2}[\eta(R(U, V) Z) X+\eta(U) R(X, V) Z \\
& +\eta(V) R(U, X) Z+\eta(Z) R(U, V) X] \\
& -S(X, R(U, V) Z) \xi-S(X, U) R(\xi, V) Z \\
& -S(X, V) R(U, \xi) Z-S(X, Z) R(U, V) \xi,
\end{aligned}
$$

taking the inner product with $\xi$ we obtain

$$
\begin{aligned}
0=( & -1) \alpha^{2}[\eta(R(U, V) Z) \eta(X)+\eta(U) \eta(R(X, V) Z) \\
& +\eta(V) \eta(R(U, X) Z)+\eta(Z) \eta(R(U, V) X)] \\
& +S(X, R(U, V) Z)-S(X, U) \eta(R(\xi, V) Z) \\
& -S(X, V) \eta(R(U, \xi) Z)-S(X, Z) \eta(R(U, V) \xi) .
\end{aligned}
$$

Putting $U=Z=\xi$ in the above equation an using (2.10)-(2.13) we get

$$
0=(n-1) \alpha^{2}\left[-2 \alpha^{2} \eta(V) \eta(X)+\alpha^{2} g(V, X)-\alpha^{2} \eta(V) \eta(X)\right]
$$




$$
+(n-1) \alpha^{4} \eta(V) \eta(X)+\alpha^{2} S(X, V)
$$

with simplify of the last equation we have

$$
S(X, V)=-(n-1) \alpha^{2} g(X, V)+2(n-1) \alpha^{2} \eta(X) \eta(V) .
$$

Therefore, in view of (2.9) manifold is an $\eta$-Einstein manifold. The proof is complete.

Definition 3.5 A Lorentzian $\alpha$-Sasakian manifold $M$ is said to be $\phi$-symmetric if

$$
\phi^{2}\left(\left(\nabla_{W} R\right)(X, Y) Z\right)=0
$$

for all vector fields $X, Y, Z, W$ on $M$ [6].

Theorem 3.6 A $\phi$-symmetric Lorentzian $\alpha$-Sasakian manifold is an $\eta$-Einstein manifold.

Proof. If manifold is $\phi$-symmetric then in view of Definition 3.5 we have

$$
\phi^{2}\left(\left(\nabla_{W} R\right)(X, Y) Z\right)=0
$$

by virtue of (2.1) we get

$$
\left(\nabla_{W} R\right)(X, Y) Z+\eta\left(\left(\nabla_{W} R\right)(X, Y) Z\right) \xi=0
$$

taking inner product with $U$, we obtain

$$
g\left(\left(\nabla_{W} R\right)(X, Y) Z, U\right)+\eta\left(\left(\nabla_{W} R\right)(X, Y) Z\right) g(\xi, U)=0 .
$$

Let $\left\{e_{i}\right\}, i=1,2, \ldots, n$, be an orthonormal basis of tangent space at any point of the manifold. Then by putting $X=U=e_{i}$ in (3.11) and taking summation over $i, 1 \leq i \leq n$, we have

$$
\left(\nabla_{W} S\right)(Y, Z)+\sum_{i=1}^{n} \eta\left(\left(\nabla_{W} R\right)\left(e_{i}, Y\right) Z\right) g\left(\xi, e_{i}\right)=0 .
$$

Replacing $Z=\xi$ in the above equation, we obtain

$$
\left(\nabla_{W} S\right)(Y, \xi)+\sum_{i=1}^{n} \eta\left(\left(\nabla_{W} R\right)\left(e_{i}, Y\right) \xi\right) g\left(\xi, e_{i}\right)=0 .
$$

The second term of (3.12), takes the form

$$
\begin{aligned}
& \eta\left(\left(\nabla_{W} R\right)\left(e_{i}, Y\right) \xi\right)=g\left(\nabla_{W} R\left(e_{i}, Y\right) \xi, \xi\right)-g\left(R\left(\nabla_{W} e_{i}, Y\right) \xi, \xi\right) \\
& -g\left(R\left(e_{i}, \nabla_{W} Y\right) \xi, \xi\right)-g\left(R\left(e_{i}, Y\right) \nabla_{W} \xi, \xi\right),
\end{aligned}
$$

with simplify of the above equation we have

$$
\eta\left(\left(\nabla_{W} R\right)\left(e_{i}, Y\right) \xi\right)=0 .
$$

The equations (3.12) and (3.13) imply that

$$
\left(\nabla_{W} S\right)(Y, \xi)=0,
$$

which gives

$$
\nabla_{W}(S(Y, \xi))-S\left(\nabla_{W} Y, \xi\right)-S\left(Y, \nabla_{W} \xi\right)=0,
$$

in view of (2.6) and (2.6) we obtain

$$
(n-1) \alpha^{2} \nabla_{W} \eta(Y)-(n-1) \alpha^{2} \eta\left(\nabla_{W} Y\right)+\alpha S(Y, \phi W)=0 .
$$

Replacing $Y$ by $\phi Y$ in (3.14) we get

$$
S(\phi Y, \phi W)=(n-1) \alpha g\left(\left(\nabla_{W} \phi\right) Y, \xi\right) .
$$

Using (2.2), (2.8) and (2.16) in the above equation we have 


$$
S(Y, W)=-(n-1) \alpha^{2} g(W, Y)-2(n-1) \alpha^{2} \eta(Y) \eta(W) .
$$

This implies that manifold is an $\eta$-Einstein.

\section{Reference}

[1] Adati, T. and Matsumoto, K., On conformally recurrent and conformally symmetric P-Sasakian manifolds, TRU Math., 13 (1977), 25-32.

[2] Boeckx, E., Buecken, P. and vanhecke, L., $\phi$-symmetric contact metric spaces, glasgow Math.J., 41 (1999) , 409-416.

[3] De, U.C., On $\phi$-symmetric Kenmotsu manifolds, International Electronic J. Math., Vol. 1, No. 1, (2009) , 33-38.

[4] De, U. C. and Sarkar, A., On $\phi$-Ricci symmetric Sasakian manifolds, Proceedings of the Janieon Math. Soc., 11 (1) (2008), 47-52.

[5] Sato, I., On a structure similar to the almost contact structure, Tensor, (N.S.), 30 (1976), 219-224.

[6] Takahashi T., Sasakian $\phi$-symmetric spaces, Tohoku Math. J. 29(1977), 91-113.

[7] Yildiz, A. and Turan, M., A class of Lorenzian $\alpha$-Sasakian manifolds, Kyungpook Math.J. 49 (2009), 789-799. 